\title{
Mobile Lunar Base Concepts
}

\author{
Marc M. Cohen
}

\author{
Advanced Projects Branch, Space Projects Division, NASA-Ames Research Center \\ Moffett Field, CA 94035-1000 \\ 650604-0068,FAX 650 604-0673,Marc.m.cohen@nasa.gov
}

\begin{abstract}
This paper describes three innovative concepts for a mobile lunar base. These concept combine design research for habitat architecture, mobility systems, habitability, radiation protection, human factors, and living and working environments on the lunar surface. The mobile lunar base presents several key advantages over conventional static base notions. These advantages concern landing zone safety, the requirement to move modules over the lunar surface, and the ability to stage mobile reconnaissance with effective systemic redundancy. All of these concerns lead to the consideration of a mobile walking habitat module and base design. The key issues involve landing zone safety, the ability to transport habitat modules across the surface, and providing reliability and redundancy to exploration traverses in pressurized vehicles. With self-ambulating lunar base modules, it will be feasible to have each module separate itself from its retro-rocket thruster unit, and walk five to ten $\mathrm{km}$ away from the $\mathrm{LZ}$ to a pre-selected site. These mobile modules can operate in an autonomous or teleoperated mode to navigate the lunar surface. At the site of the base, the mobile modules can combine together; make pressure port connections among themselves, to create a multi-module pressurized lunar base.
\end{abstract}

\section{INTRODUCTION}

The mobile lunar base presents several advantages over conventional static base notions. These advantages concern landing zone safety, the requirement to move modules over the lunar surface, and the ability to stage mobile reconnaissance with effective systemic redundancy. All of these concerns lead to the consideration of a mobile habitat module and base design. The key issues that the design of a mobile base must resolve include automated assembly and deployment, the configuration and methods for connecting modules, radiation protection and mission duration. The principal advantage of the stationary base is that it affords the build-up of infrastructure and resources in one location, which can lead to economies of scale and of agglomeration, without added the transportation costs of making it all mobile. One leading prospect of infrastructure build-up is to provide radiation shielding by burying the modules under 1 meter or more of lunar regolith.

\section{Landing Zone}

The landing zone (LZ) poses the problem that once a habitat lands on the moon, it is not prudent to land another vehicle within several kilometers because of safety concerns from ejecta in a normal landing and in case of an explosive failure on impact. Therefore, if the lunar mission intends to create a well-established approach range and LZ, it is necessary to move the landed habitats and payloads well away from the LZ, much in the manner that aircraft taxi off the runway after landing at an airport. So what is the best way to move the module?

\section{Exploration Mobility Approaches}

A further advantage of the mobile lunar base concerns exploration traverses of the lunar surface. The conventional model of exploration is that a crew of two or more astronauts travel in a pressurized or unpressurized rover to a remote site, perform an EVA, collect some rock, soil and regolith samples, and then return to the base. An unpressurized rover is limited to a traverse measured in hours. If the rover is pressurized, then the crew can make a longer traverse, under some scenarios lasting days (sols) or weeks. 


\section{Reliability, Redundancy and Availability}

The problem with this conventional rover scenario is one of reliability and redundancy. If the rover should experience a failure that prevents its return to base or that otherwise compromises safety or is life threatening, how will other astronauts at the lunar base rescue them? The usual answer is to have a second rover that can perform the rescue. But what if it runs into a problem too - the same or a different problem? Well, that means a third rover. Following this chain of reasoning, fairly soon, most or all of the landed mass resources of the lunar mission becomes devoted to ensuring the safety of a pressurized traverse mission.

So, why not make the entire base mobile, so that all the resources, reliability and redundancy of the lunar mission move with the exploration crew? This approach means that the laboratory facility will travel with the explorers, affording them the capability to conduct complex and sophisticated scientific assays and analyses on site, without a need to return to the base. Once the lunar exploration concept transcends the convention of a rover as a delivery truck for rocks, vast new potentialities open up. The Mobile Lunar base allows the explorers to bring the base to the sites of scientific interest to make the most complete investigation, without the severe constraints and limitations to traverses and EVA sortie time. A further advantage of the Mobile Base system is availability. With the versatility of the Mobile Base approach, it is possible to land new mobile modules with new equipment, supplies or logistical support in the path of the moving ensemble. These new units could then join the "wagon train" to continue on the journey, or simply provide a cache of supplies for the crew to pick up along the way (Cohen, 2003).

\section{"WAGON TRAIN" CONCEPTS}

The "Wagon Train" was an American pioneering technique in which a group of ox-drawn Conestoga wagons traveled together in line. When they stopped for the night, they would "circle the wagons" to form a temporary base cluster. Three concepts draw upon various aspects of the wagon train paradigm: the Lunar Rover Complex, the Habot and the Mobitat.

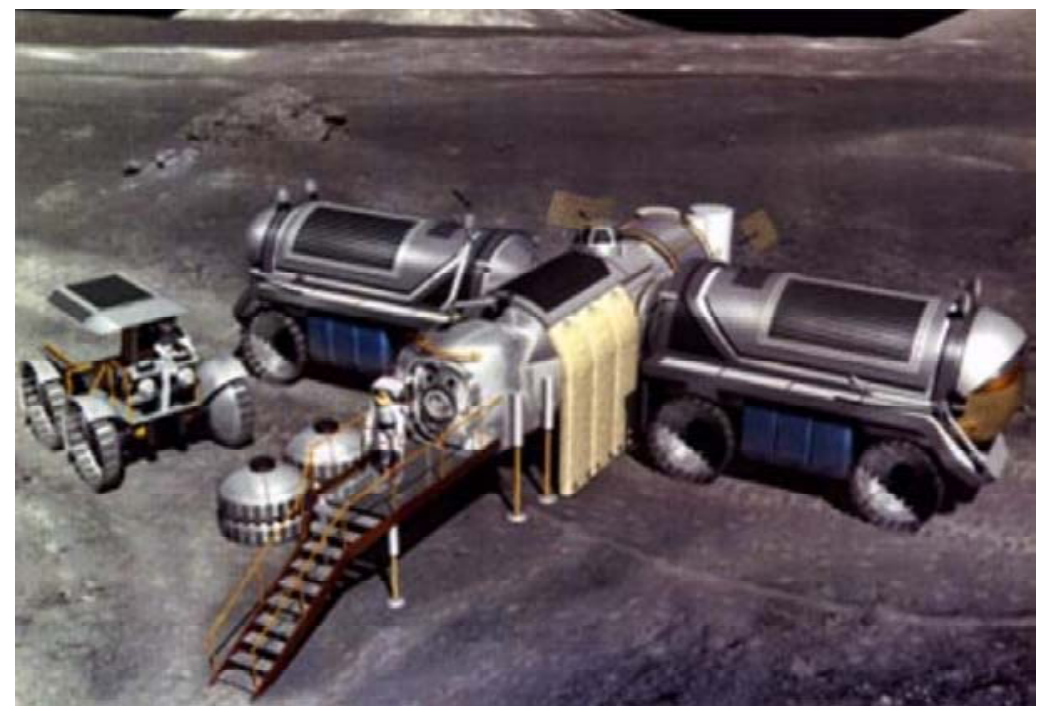

FIGURE 1. Frassanito's Lunar Rover Base complex, 1993 (Courtesy of NASA-Johnson Space Center).

\section{Frassanito's Lunar Rover Complex, 1993}

The industrial designer John Frassanito provided many of the design images for the NASA Mars Design Reference Mission (Hoffman, Kaplan, 1997), and the studies that lead up to it (Weaver, Duke, 1993). One offshoot of these studies was a concept for a lunar base formed by a group of several independent lunar rovers. In this concept, the 
rovers would travel together as individual units "wagon train" style. At a likely site they would join together to form a temporary base. FIGURE 1 illustrates this concept. There is a central "core module" with two lateral docking ports on either side. At the end showing in the picture is an EVA airlock exiting to a deployable platform and stair. The driver's position is presumably at the distal end of the core module, although perhaps it could be towed. The two modules docked on each side of the core would "back into" the docking ports. The driver's station appears on the right as a large gold-coated lens. While the pressurized modules are docked together, they afford a continuous atmosphere among the three vehicles. While the rovers are docked, the crew would employ the small, unpressurized rover on the left for local mobility.

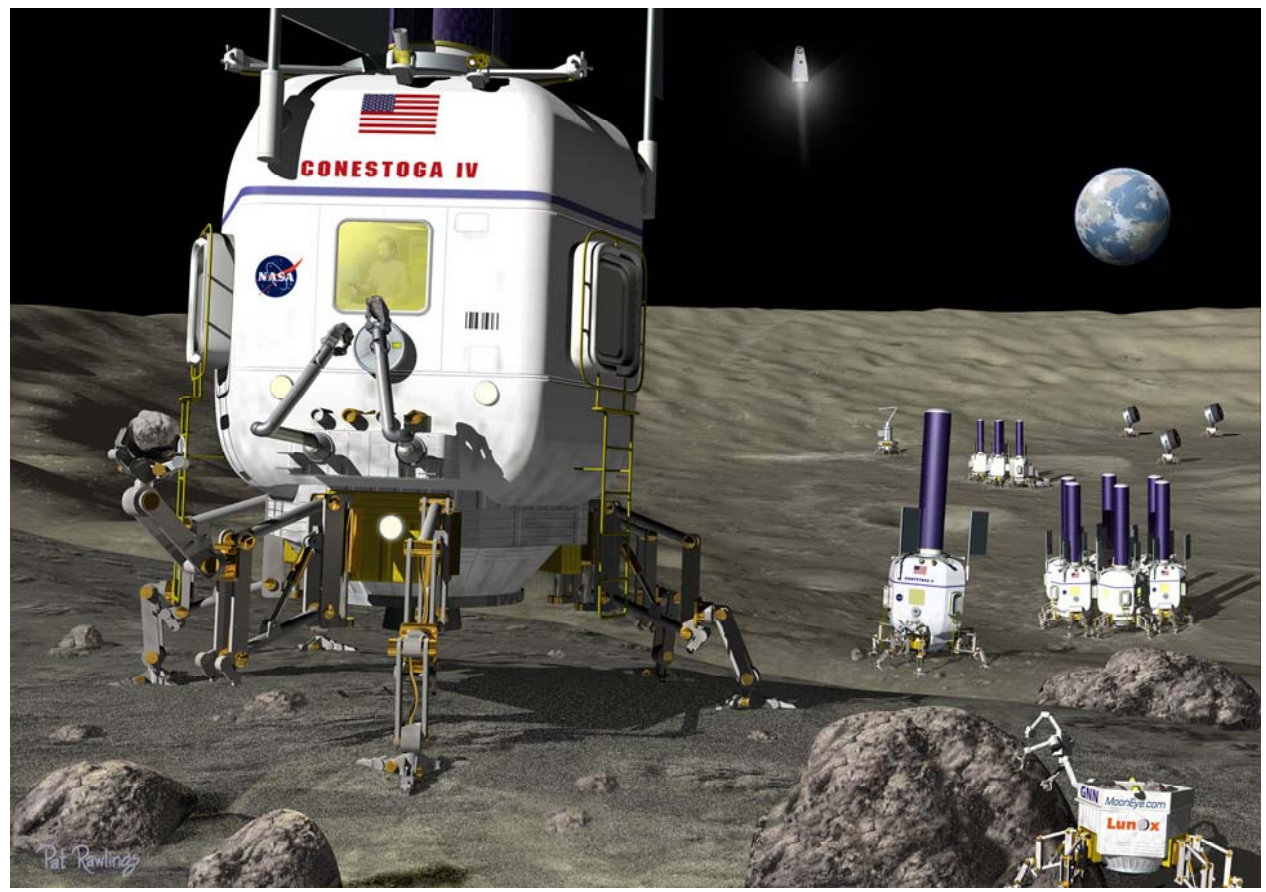

FIGURE 2. Pat Rawlings' Rendering of the "Habot" Mobile Lunar Base Concept (courtesy of John Mankins, NASA HQ, and Neville Marzwell, Jet Propulsion Laboratory).

\section{Mankins' Habot}

John Mankins introduced the Habitat Robot concept, "Habot," in 2000 as a radical departure from traditional lunar base studies. The strongest attribute of the Habot is its "small is beautiful" emphasis. The Habot modules land on six articulated legs, and then takes double advantage of the legs by using them to walk away robotically from the landing zone. The pressure vessels are hexagonal insofar as they require three cross-axes separated equally at $60^{\circ}$ of arc. They cluster together automatically to form a temporary base. The possible module size ranges from about 3 to $5 \mathrm{~m}$ diameter. The crew arrive and depart the surface of the moon in a separate Descent/Ascent vehicle that may share some hardware commonality with the Habot units, but is optimized for transporting the crew through cislunar space, landing and taking off from the lunar surface. With self-ambulating lunar base modules, it would be feasible to have each module separate itself from its retro-rocket thruster unit, and walk five to ten $\mathrm{km}$ away from the LZ to a pre-selected site. These walking modules can operate in an autonomous or teleoperated mode to navigate the lunar surface. At the site of the base, the walking modules can combine together; make pressure port connections among themselves, to create a multi-module pressurized lunar base. FIGURE 2 presents an artist's rendering of the Habot concept, showing both a hexagonal Habot cluster base, and Habot rovers moving about the lunar surface, driven by astronauts. A peculiar power source appears in the form of the cylinders mounted atop each Habot, which mount photovoltaic cells to provide power during the 14 sol lunar day. With exposed joints, the articulated legs would be vulnerable to dust and grit in the mechanisms, so the legs would need to be covered with dust-tight "stockings" 


\section{Lai and Howe's Mobitat}

Inspired by the Habot concept, Prof. A. Scott Howe and his student Lai Yip Hung at the University of Hong Kong conceived of the Mobitat as a way to integrate existing automated and robotic construction technologies with a lunar surface habitat (Lai and Howe, 2003). In the Mobitat scheme, each habitat module lands with a kind of integrated gantry crane. This "crane" carries the descent rocket engines. FIGURE 3 shows a Mobitat folded in descent configuration. The crane transports the habitat module; deposits it at the mobile base site and can assemble a cluster of the habitats. FIGURE 4 shows the Mobitat in deployed configuration for movement on the lunar surface. One crane could carry many pressure vessel units to the base. However, since each Mobitat lands with an integrated crane, this strategy might soon lead to a surplus of mobile cranes without their pressure vessel units. Although this crane is designed to handle extremely rough terrain, it is probably too massive relative to the habitat pressure vessel to fulfill the Habot concept. It is probably too large even to allow the berthing together of a ring of habitats while still attached to the habitats. However, it should be possible to redesign this schematic for the mobile crane to be smaller, lower and to drop the descent engines after landing.

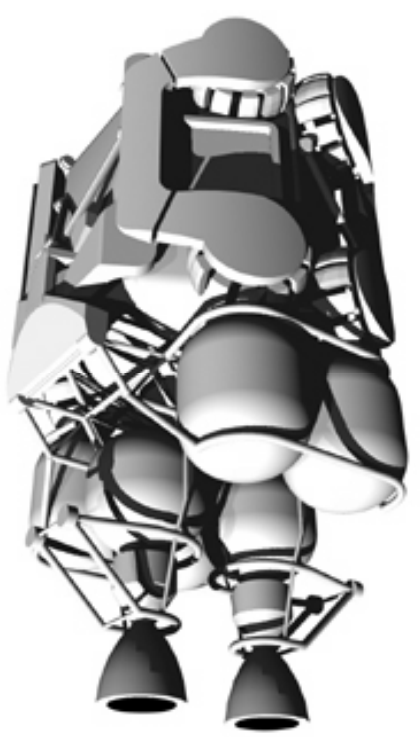

FIGURE 3. Mobitat Folded in Descent Configuration (by permission of A. Scott Howe).

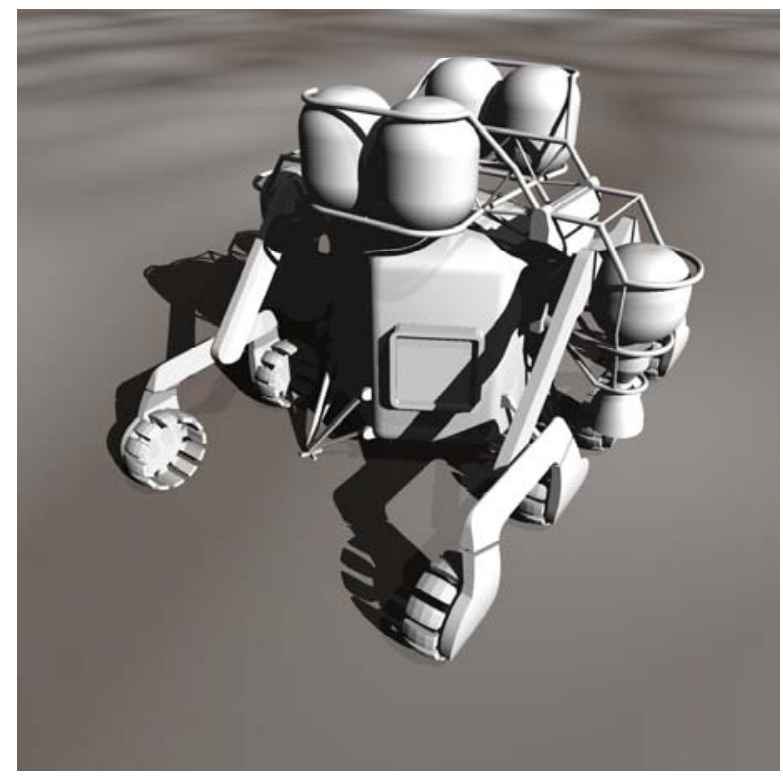

FIGURE 4. Mobitat Deployed to Transport Habitat Module (by permission of A. Scott Howe).

\section{Discussion}

Each of these three concepts for "Wagon Train" mobile lunar bases presents important advantages and disadvantages. Each, in its own way, points to a particular design strategy, all of them valid within certain parameters and requirements. Frassanito's Lunar Rover Complex's strength is that it makes the best use of "existing" technologies for wheeled vehicles. Frassanito's major weakness is that the ability to expand the mobile base is limited generally by the geometry and dimensions of the rovers themselves - the number of berthing ports are proportional to the length of the central berthing and EVA module. Mankin's Habot's strength lies in making the best use of the articulated legs that are essential for a controlled, soft landing. The Habot's weakness is that such a hexagonal plan, hexapod walking system does not appear to exist yet, and if it did, it would have serious issues of keeping the pressure vessel level and stable while walking. Lai and Howe's Mobitat offers the advantage of the most advanced thinking about integrating the mobility system with the mobile habitat through all phases of the mission. However, the Mobitat's weakness is that to achieve this integration and concomitant flexibility, the mobility system is much too big. Beyond the major strengths and weaknesses each of these three mobile lunar base concepts present interesting design ideas and even details that shall be the subject of further analysis. 


\section{KEY HABOT PARAMETERS}

The fundamental idea is that the Habot is a combination of a human habitat and a robot. The Habot lands autonomously on the Moon on a set of articulated legs. The Habot then uses those legs or wheels mounted on the legs to move itself away from the designated landing zone so that more Habots can land. Once enough Habots arrive to form the lunar base, they cluster together at a site of scientific or technical interest, and make the vital connections for pressurized access.

\section{Robotic Habitat Deployment and Verification}

The basic issue that the Habot addresses -- more than any of the previous concepts - is how to best use the cost and effort of very expensive crew time on the lunar or planetary surface. Gordon Woodcock of Boeing led a notable study on the use of lunar surface robotics that took into consideration what were the best uses for humans and for robots (Woodcock et al., 1990). Race, Criswell and Rummel (2003) posed the question this way: "Can a habitat be deployed or built robotically on the surface and its operational readiness be fully verified prior to sending humans there?"

Once enough mobile base units arrive to form the lunar base, they cluster together at a site of scientific or technical interest, and make the vital connections for pressurized access, communications, data, life support, etc. After these modules have completed joining together to form the lunar base, it becomes possible for the first lunar expedition crew to arrive. After the crew completes their mission at that particular site, they return to the Earth, in a separate, dedicated vehicle. In the following weeks or months, the mobile units separate from one another, and move across the lunar surface to a new location of scientific interest, and a second crew arrives. It is also possible for the crew to travel with the mobile base units. The crewmembers will also use individual mobile units as pressurized rovers to explore the lunar environment. In FIGURE 2 the articulated legs carry manipulator devices that can pick up rocks. A hexagonal cluster appears in the middle ground at the right.

\section{Mission Activities}

John Mankins $(2000,2001)$ describes the character of a mobile lunar base mission. In his "notional" concept, the Habot mission baseline would be 100 sols with the capability of supporting six crewmembers for that duration. (where the term sol refers to a 24 hour Earth day, such that a lunar day would be 28 sols). However, the analysis for this section takes a different approach. Within the baseline 100 days, the nominal Habot mission would for a crew of four astronauts to spend two complete lunar day/ night cycles - 56 sols - on the lunar surface, with a planned 8sol margin for a total planned mission duration range of 64 sols. The 36 additional sols would constitute a reserve capacity. In Mankins' construct, the Habot infrastructure should serve a baseline of 10 crews rotating through the Habot base, each time in a different location on the lunar surface, for a total of 1000 sols of occupancy, which approaches the overall time necessary for a human Mars mission. This baseline implies a total productive occupancy of 560 sols with a total planned margin of 80 sols. Total reserve would be 360 sols. Of course, in the event that the crew had a problem lifting off from the Moon, it would be possible to resupply them from the Earth, almost anywhere on the moon.

This initial phase of the Mobile Lunar Base study is considering crew sizes from 2 to 8 crewmembers for the purpose of assessing the relationship between crew size and productivity. These crewmembers would occupy and utilize several mobile modules. The lesson of the International Space Station is quite dramatic: with three crew members on board, they spend almost all their time just maintaining and operating the station, with very little time minutes per day, really - to perform science. The Mobile Lunar Base study includes a detailed study of crew time for this range of crew sizes to analyze the affects of the skill mix and required "overhead" activities upon prospective crew productivity. During the lunar day, the crew will conduct the exploration portion of the mission. This study relies upon Nash et al. (1989) to provide a baseline for exploration capabilities required. During the lunar day, the Habot units will make maximum utility of their walking capability. Thus the Habot units will move separately across the lunar terrain, meeting and docking as necessary for various crew operations and procedures. As the lunar day approaches its end, the Habots will cluster together and dock, creating a continuous pressurized 
habitable environment. During the lunar night, the crew will stay primarily in this united lunar base, and pursue work that they can perform in the laboratories.

\section{Preliminary Mass Budget}

The original concept for the Habot aimed for a mass budget per unit of 3 to 5 mTons. This mass limit would be very convenient for launch by existing conventional expendable rockets. However, as a preliminary analysis the $5 \mathrm{mTon}$ mass budget per unit has small margin and overall is extremely tight for a nominal 100-sol mission by the crew of 4 . A more realistic Habot mass budget baseline may be closer to $10 \mathrm{mTon}(10,000 \mathrm{~kg})$, separate from the descent engine unit. TABLE 1 presents a preliminary mass budget for this Habot unit, working with the range of masses that Mankins envisions. These bounding values appear in the top line for the pressurized habitat and its contents, including outfitting. However, these mass values are simply too small to provide the complete system for one Habot with a crew of 4 over 100 days. The lines below the pressurized habitat indicate the additional elements that would be needed.

TABLE 1. Preliminary Habot Mass Budget.

\begin{tabular}{|c|c|c|c|}
\hline Component & Min Mass, mTon & Max Mass, mTon & Remarks \\
\hline $\begin{array}{l}\text { Pressurized Habitat: } \\
\text { Pressure Vessel Structure, Life Support \& } \\
\text { Thermal Control, Habitability } \\
\text { Accommodations, Operational Systems }\end{array}$ & 3.0 & 5.0 & $\begin{array}{l}\text { Habitat is fully pre- } \\
\text { integrated before } \\
\text { launch. }\end{array}$ \\
\hline $\begin{array}{l}\text { Exterior Protection } \\
\text { Radiation Shielding, Thermal Protection, } \\
\text { Micrometeoroid Protection }\end{array}$ & 2.0 & 2.8 & $\begin{array}{l}\text { Exterior protection is a } \\
\text { multi-function system. }\end{array}$ \\
\hline $\begin{array}{l}\text { Mobility System } \\
\text { Habot "Unibody" consisting of Base frame, } 6 \\
\text { legs, motors and mechanisms. }\end{array}$ & .8 & 1.0 & $\begin{array}{l}\text { Mobility system } \\
\text { requires much further } \\
\text { study. }\end{array}$ \\
\hline $\begin{array}{l}\text { Energy Systems } \\
\text { Solar Cells, batteries, SSP/"Powerbot" } \\
\text { Microwave Antenna, Possible RTG }\end{array}$ & .5 & .8 & $\begin{array}{l}\text { Energy system requires } \\
\text { a system and } \\
\text { optimization study. }\end{array}$ \\
\hline Margin & 1.0 & .4 & \\
\hline Limits (not totals) & 7.3 & 10.0 & \\
\hline
\end{tabular}

This analysis leads away from the traditional approach to the mass and capacity question: How many crewmembers can this Habot support and sustain on the lunar mission? Because of the modularity of the Habot system it leads to a different formulation of the question: How many Habots will be necessary to support the required crew? The next question is: What is the optimal distribution of equipment, supplies and mass among these several Habot units? The answer to this second question will demand a very detailed exercise in design optimization.

\section{Energy System}

The energy system incorporates several elements. In FIGURE 2 showing the Habot, a cylindrical tower atop the module carries photovoltaic cells to provide constant "lifeline" power during the lunar day. Atop this tower sits a parabolic dish antenna to receive beamed microwave or laser power. A possible back-up option for "lifeline" power would be to install a radio-thermal generator (RTG) at the base of the tower. Safe disposal of spent nuclear fuel will be required to make this concept viable. However, providing sufficient and reliable energy throughout the lunar day/night cycle remains one of the technological "tall poles" to make any mobile base concept succeed, and will demand much more research and development. In addition to the Habot approaches to energy systems are two possible alternatives using beamed energy, proposed by Williams, et al., and by Cataldo. 


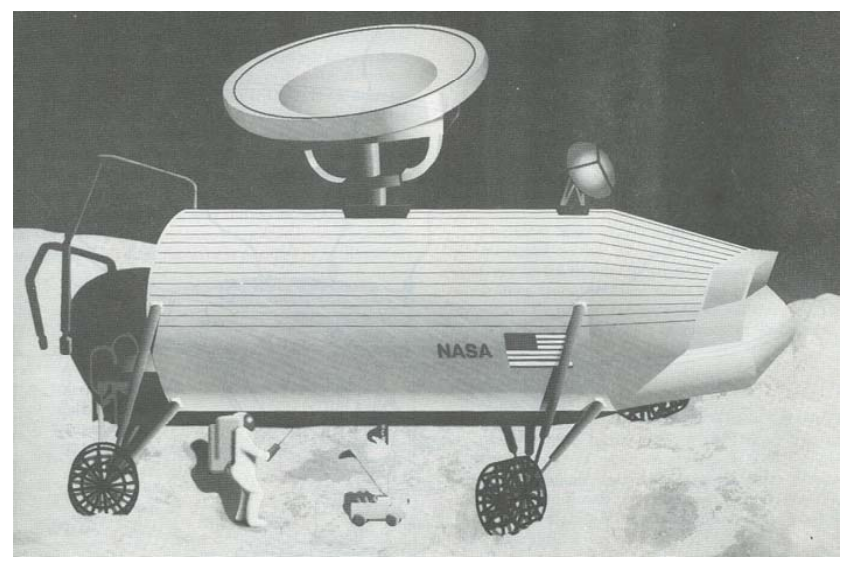

FIGURE 5. Williams et al.'s concept for a lunar rover powered by space solar power satellite via laser beam to the dish antenna on top (Courtesy of NASA-Langley Research Center).

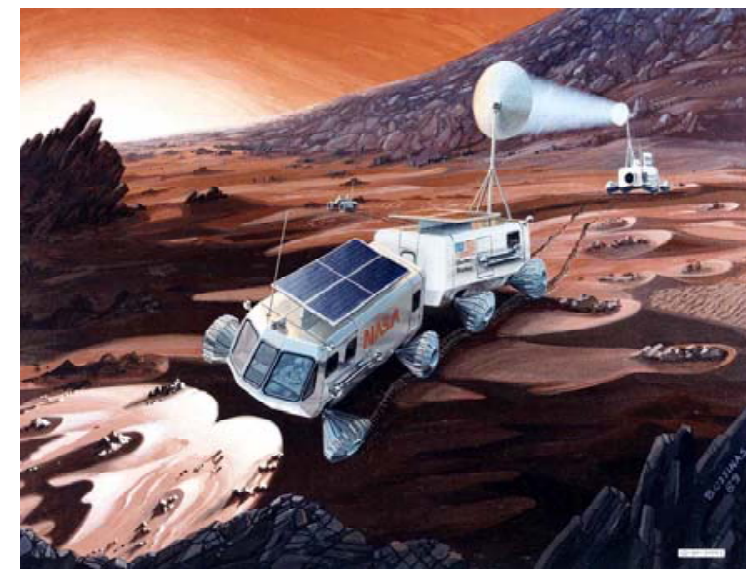

FIGURE 6. Robert Cataldo's concept for a "follower" nuclear reactor rover (courtesy of Robert Cataldo, NASAGlenn Research Center).

Williams et al. (1993) proposed such a scheme to power a lunar rover by beaming laser power from a solar power satellite to a parabolic receiver on a pressurized rover. The primary source of this power will be a space solar power satellite in a lunar-synchronous orbit that would keep the satellite above the rover at all times which would provide power in the 100 to $300 \mathrm{KW}$ range. However, it is not clear how it would supply power when in the shadow of the moon. Such a concept might be more viable in combination with solar power satellites at the lunar L1 or L2 point or both, The Williams concept appears in FIGURE 5. The large laser beam antenna is mounted in the center of the cylindrical portion and the small antenna to the front is for communications.

A second power alternative would be a nuclear reactor (Cataldo) mounted on a Habot, Rover or Mobitat chassis FIGURE 6 shows Robert Cataldo's mobile Lunar Reactor concept, following and powering a pressurized rover. This "Powerbot" would follow the Habots from a distance of several kilometers away, and beam power in the 100 to 150 $\mathrm{KW}$ range by microwave to the same antenna that would serve for solar satellite power.

\section{Mobility System}

This study does not presuppose any specific mobility system. Only after analyzing all of the necessary functions and components of the mobile habitat and base configurations, will it be reasonable to develop requirements for the mobility system. Never the less, since the Habot is closely associated with the walking model, it is appropriate to describe the candidate walking aspect. The Habot will have a very modest walking speed that need not exceed 5 $\mathrm{km} /$ hour. There is no advantage in designing it to move "fast" if that translates into a huge energy burden that will be used for only short periods of time. The baseline is a maximum of $2 \mathrm{~km} / \mathrm{hr}$ with a crew driver over smooth, level terrain. On rough terrain, the speed will be reduced to whatever is safe, perhaps as slow as $.5 \mathrm{~km} / \mathrm{hr}$ on slopes or rough terrain. The baseline speed without a crew on board is $.5 \mathrm{~km} / \mathrm{hr}$.

All Habots will land uncrewed. They will walk or roll themselves about 10km away from the LZ to a base deployment site. There, the Habots will dock together and await the arrival of the crew. When the crew land in the descent/ascent vehicle, they travel on the same walking system to the base deployment site. There, the crew transfers via a docking tunnel in a shirtsleeve environment to the united base. As a contingency, the descent/ascent vehicle will carry EVA suits the crew can use to make the transfer. Additional contingencies if the descent/ascent vehicle is unable to walk, a Habot from the base will come to the LZ and pick up the crew. The final fallback is that the crew can walk the $10 \mathrm{~km}$ EVA to the base. 
TABLE 2. Habot Module Types.

\begin{tabular}{|l|l|}
\hline Living Environment/Habitability Support & Working Environment \& Operational Systems \\
\hline - Crew "Cabin" (Sleeping Quarters). & $\begin{array}{l}\text { - EVA Access: Airlock and Stowage, including the } \\
\text { NASA “Robonaut" anthropomorphic master-slave tele- } \\
\text { robot and EVA suit Stowage and maintenance. }\end{array}$ \\
\hline $\begin{array}{l}\text { - Ward Room (back-up Command and } \\
\text { Communications Center). }\end{array}$ & \begin{tabular}{l} 
- Command and Communications Center. \\
\hline - Logistics Module
\end{tabular} \\
\hline - Health Maintenance, Exercise, Recreation & $\begin{array}{l}\text { - Laboratory\# 1, Life Sciences. } \\
\text { Observatory). }\end{array}$ \\
\hline
\end{tabular}

\section{Habot Module Types}

In his 2000 and 2001 articles, Mankins proposes six Habot modules that form the Modular Integrated Lunar Outpost "MILO" cluster. These modules would all derive from the same basic pressure vessel, platform and chassis, comprising the complete living and working environment: The allocation of functions to this concept is somewhat reminiscent of the early stages of the Space Station, when NASA was proposing two US habitability modules and two US lab modules, plus an airlock. TABLE 2 shows the way in which the module types are being interpreted for this study, resulting in eight types of Habot base unit.

\section{CONCLUSION}

This article reviewed three mobile base concepts, and found that each offers particular strengths and weaknesses. Further study of these design ideas and their details will be instructive for formulating an optimal approach to the Mobile Lunar Base construct. The most significant development is the reliance upon automation and robotics assembly to move the mobile units across the lunar terrain and then to assemble them and verify the readiness of the base for the arrival of the crew. A major challenge emerges as allocation of resources and distribution of capabilities among the Habot modules. Radiation protection remains a challenge and a potential showstopper, as it does for all Lunar Base and rover concepts. The energy systems stand out as a technological tall pole for all mobile base concepts. The architecture for combining the pressurized modules - the habitats -- into the base cluster will play a substantive role in facilitating the use of resources and application of capabilities of all types.

\section{NOMENCLATURE}

AIAA = American Institute of Aeronautics and Astronautics

EVA = Extravehicular Activity

Habot = "Habitat Robot"

IAF = International Astronautical Federation (Congress)

ICES = International Conference on Environmental Systems

$\mathrm{KW}=$ Kilowatt

L1, L2 = Earth-Moon libration points

LEO = Low Earth Orbit

$\mathrm{LZ}=$ Landing Zone

MILO = Modular Integrated Lunar Outpost cluster

Mobitat $=$ Mobile Habitat

mTon = Metric Ton, 1,000 kilograms

RTG = Radio-thermal generator

SAE $=$ Society of Automotive Engineers

Sol $=$ One solar Earth day, 24 hours 


\section{ACKNOWLEDGMENTS}

I wish to thank Tony Gross, NASA-Ames; John Mankins, NASA-HQ; Neville Marzwell, NASA-JPL; and Thomas Wynn, NASA-Ames for their generous support and encouragement to pursue this analysis.

\section{REFERENCES}

Cohen, Marc M., "Mobile Lunar and Planetary Bases," AIAA-2003-6280, AIAA Space 2003 Conference, San Diego, CA, Sept. 23-25, 2003. Reston VA, AIAA, 2003.

Hoffman, Stephen J., and Kaplan, David I., eds, Human Exploration of Mar: The Reference Mission of the NASA Mars Exploration Study Team, NASA SP-6107, Houston, TX, NASA- Johnson Space Center, 1997.

Lai, A. Yip Hung; Howe, A. Scott, "A Kit -of-parts Approach to Pressure Vessels for Planetary Surface Construction," AIAA2003-6281, AIAA Space 2003 Conference, San Diego, CA, Sept. 23-25, 2003. Reston VA, AIAA, 2003.

Mankins, John C., "Modular Architecture Options for Lunar Exploration and Development," IAA.13.2.05, 51 ${ }^{\text {st }}$ International Astronautical Congress, Rio de Janeiro, Brazil. October 2-6, 2000, Paris, France, IAA.

Mankins, John C., "Modular Architecture Options for Lunar Exploration and Development," Space Technology, 21, pp. 53-64, 2001.

Nash, Douglas B., Plescia, Jeffrey, Cintala, Mark, Levine, Joel, Lowman, Paul, Mancinelli, Rocco, Mendell, Wendell, Stoker, Carol, Suess, Steven, Science Exploration Opportunities for Manned Missions to the Moon, Mars, Phobos, and an Asteroid, NASA Office of Exploration Doc. No. Z-1.3-001, JPL Publication 89-29, Washington DC, NASA Office of Exploration, 1989.

Race, Margaret S., Criswell, Marvin E., Rummel, John D., "Planetary Protection Issues in the Human Exploration of Mars," SAE 2003-01-2523, 33 ${ }^{\text {rd }}$ ICES, Vancouver, BC, July 11-14, 2003, Warrendale, PA, SAE, 2003.

Weaver, David B. \& Duke Michael B. "Mars Exploration Strategies, A Reference Program and Comparison of Alternative Architectures," AIAA 93-4212, AIAA Space Programs and Technologies Conference and Exhibit, Huntsville, AL, September 21-23, 1993, Reston VA, American Institute of Aeronautics and Astronautics, 1993.

Williams, M. D., De Young, R. J., Schuster, G. L., Choi, S. H., Dagle, J. E., Coomes, E. P., Antoniak, Z. I., Bamberger, J. A., Bates, J. M., Chiu, M. A., Dodge, R. E., and Wise, J. A., Power Transmission by Laser Beam from Lunar- Synchronous Satellite, NASA TM 4496, pp. 19-20, Washington DC, National Aeronautics and Space Administration, 1993.

Woodcock, Gordon R., et al. Robotic Lunar Surface Operations, Boeing Report D 615-11901, NASA Contract No. NAS 212108, Huntsville AL, Boeing Aerospace \& Electronics Company, 1990. 